\title{
LA TRASCENDENCIA DE LA COMUNICACIÓN INTERGENERACIONAL EN LA FORMACIÓN DE LOS MENORES \\ EL PAPEL DE LOS ABUELOS EN LAS SOCIEDADES OCCIDENTALES DE NUESTRO TIEMPO
}

\section{THE IMPORTANCE OF INTERGENERATIONAL COMMUNICATION IN THE EDUCATION OF MINORS \\ THE ROLE OF GRANDPARENTS IN TODAY'S WESTERN SOCIETIES}

\begin{abstract}
Manuel Carmona Rodríguez ${ }^{a^{*}}$
\end{abstract}
Fechas de recepción y aceptación: 22 de junio de 2021 y 21 de octubre de 2021

DOI: https://doi.org/10.46583/scio_2021.21.916

Resumen: Las familias españolas y occidentales tienen problemas en la comunicación diaria. Esa incomunicación también se da en los distintos niveles del sistema educativo: la ESO, el Bachillerato, la FP y la Universidad. Esto afecta a nuestra juventud entre los 13 y 18 años, que, salvo ejemplos excepcionales, vive desorientada sobre qué quiere hacer con su vida. A pesar de la existencia de orientadores en los centros educativos, la escasez de recursos humanos en esa tarea, unida al obsesivo objetivo por aprobar cursos y la EvAU, está lastrando la formación y orientación de nuestra juventud. Preguntas decisivas que todos nos hacemos a lo largo de la vida $u$ otras que afectan a las relaciones interpersonales, intergeneracionales y sociales no se abordan de frente porque las personas, las familias, los centros educativos, desde los institutos hasta las universidades, y las instituciones administrativas y políticas las están obviando. En este artículo se recurre a las experiencias de vida para reflejar nuestro tiempo, y la necesidad de mejorar los hábitos cotidianos practicando una educación interdisciplinar en formación vocacional,

a Departamento de Periodismo y Comunicación Corporativa. Universidad Rey Juan Carlos

* Correspondencia: Universidad Rey Juan Carlos. Departamento de Periodismo y Comunicación Corporativa. Campus de Fuenlabrada. Camino del Molino, 5, 28942 Fuenlabrada (Madrid). España.

E-mail: manuel.carmona@urjc.es 
inteligencia emocional y sentimental, educación cívica y moral, formación histórica, instrucción jurídica, educación intergeneracional o en la mirada crítica sobre los medios de comunicación y las redes sociales. La metodología aplicada será la filosofía de la razón vital e histórica y la teoría de las generaciones de Ortega y Gasset y de Julián Marías (Marías, 1961: 1). Además, se recurre a estudios nacionales e internacionales para aportar información, conocimiento y análisis clarificadores.

Palabras clave: Comunicación, intergeneracional, familia, vocación, educación interdisciplinar, coherencia.

Abstract: Spanish and Western families have problems in daily communication. This lack of communication also occurs at the different levels of the educational system: ESO, Bachillerato, FP and University. This affects our youth between the ages of 13 and 18, who, except for exceptional examples, live disoriented about what they want to do with their lives. Despite the existence of guidance counselors in educational centers, the scarcity of human resources in this task, together with the obsessive objective of passing courses and the EvAU, is weighing down the training and guidance of our youth. Decisive questions that we all ask ourselves throughout our lives or others that affect interpersonal, intergenerational and social relationships are not being addressed headon because individuals, families, educational centers from high schools to universities, and administrative and political institutions are ignoring them. In this article, life experiences are used to reflect our times, and the need to improve daily habits by practicing an interdisciplinary education in vocational training, emotional and sentimental intelligence, civic and moral education, historical training, legal instruction, intergenerational education or a critical look at the media and social networks. The methodology applied will be the philosophy of vital and historical reason and the theory of generations of Ortega y Gasset and Julian Marias (Marias, 1961: 1). In addition, national and international studies will be used to provide information, knowledge and clarifying analysis.

Keywords: Communication, intergenerational, family, vocation, interdisciplinary education, consistency. 


\section{§1. OBJetivos}

El objetivo principal de este artículo es presentar las carencias que las sociedades española, europea y occidental han desarrollado desde los años ochenta hasta el presente en la comunicación intergeneracional, que afecta a la formación humana, académica, profesional y cívica de la juventud. Esas lagunas están provocando la desorientación de los nacidos entre 1998 y 2012, la generación que en la actualidad tiene entre 22 años, los mayores, y 8 años, los más pequeños. Y de continuar así, también afectará a la siguiente.

Tengamos presente, siguiendo a Ortega, que anunció que toda nueva generación se da de alta en la sociedad a partir de los 30 años, que esos jóvenes presentarán al mundo su modelo de vida, de sociedad y de país hacia 2028. En palabras de Ortega:

Los cambios históricos suponen el nacimiento de un tipo de hombre distinto en más o en menos del que había; es decir, suponen el cambio de generaciones. El concepto de generación es el más importante en historia. Para que algo importante cambie en el mundo es preciso que cambie el tipo de hombre y el de mujer; es preciso que aparezcan muchedumbres de criaturas con una sensibilidad distinta de la antigua y homogénea entre sí. Esto es la generación: una variedad humana en el sentido riguroso que al concepto de variedad dan los naturalistas (...) Hoy es para unos 20 años, para otros cuarenta, para otros sesenta; y eso, que siendo tres modos de vida tan distintos, tengan que ser el mismo hoy, declara sobradamente el dinámico dramatismo, el conflicto y colisión que constituye el fondo de la materia histórica, de toda convivencia actual. Todos somos contemporáneos, vivimos en el mismo tiempo y atmósfera, pero contribuimos a formarnos en tiempo diferente. Solo se coincide con los coetáneos (Ortega, 1929, pp. 289-290).

Pero esos problemas de incomunicación, de falta de comprensión y entendimiento, de no ponerse en la piel del otro y en sus circunstancias, también se están dando en las generaciones de adultos, aquellas que nacieron en los años 1930, 1945, 1960, 1974 y 1998. Todas ellas, junto a la de los menores, son las generaciones que en la actualidad conviven. 
Esas lagunas de comunicación también son consecuencias de la debilidad de los usos, de las vigencias y creencias en las circunstancias familiares, sociales, educativas e institucionales. Hay mucha volatilidad y, por tanto, nuestros jóvenes no saben a qué atenerse, pero es que muchos adultos tampoco. A lo largo de este artículo se van a ir presentando esos problemas de la vida familiar con una trascendencia que alcanza a la vida social: el descubrimiento de la vocación y de la vida personal; el envejecimiento de la sociedad occidental; la falta de una buena educación alimentaria; los extremismos que vuelven de la mano de los movimientos nacionalistas excluyentes y la necesidad de una formación histórica sólida para darles respuesta; el valor terapéutico de la narrativa; la educación orientada a analizar de forma crítica los medios de comunicación; la formación de padres y madres; y la educación emocional y sentimental.

\section{§2. PRoblemas DE LA VIDA FAMILIAR: UNA CUESTIÓN VITAL}

Ortega, en su ensayo El hombre y la gente, realiza un hallazgo clave para entender el paso o la circunstancia que hay entre la vida personal y la vida social. Se detiene a definir y describir las relaciones interpersonales. El primer contexto en que esas relaciones interpersonales se desarrollan es la familia. En palabras de Ortega:

Lo social es un hecho, no de la vida humana, sino algo que surge en la humana convivencia. Por convivencia entendemos la relación o trato entre dos vidas individuales. Lo que llamamos padres e hijos, amantes, amigos, por ejemplo, son formas del convivir. En ellas se trata siempre de que un individuo, como tal -por tanto, un sujeto creador y responsable de sus acciones, que hace lo que hace porque tiene para él sentido y lo entiende-, actúa sobre otro individuo que tiene los mismos caracteres. El padre, como individuo determinado que es, se dirige a su hijo, que es otro individuo determinado y único también. Los hechos de convivencia no son, pues, por sí mismos hechos sociales. Forman lo que debiera llamarse «compañía o comunicación»-un mundo de relaciones interindividuales-(Ortega, 1957: 4). 


\subsection{El envejecimiento de la sociedad europea y occidental}

Entre los retos que afrontan la Unión Europea y Occidente, dado el envejecimiento presente y futuro de su población, está el retomar la senda de los programas europeos y trasatlánticos que apoyen de forma cualitativa y decisiva la inclusión social, sobre todo tras los recortes económicos sufridos entre el decenio de 2008 a 2018. En buena medida no solo el mañana de la civilización europea y occidental está en juego, sino también el propio destino de esta civilización y de sus relaciones con los pueblos asiáticos y africanos.

Las cuestiones vinculadas con la Educación, la Salud y la Atención Sanitaria entendidas desde un prisma que abarque todas las circunstancias de la vida en el sentido filosófico de Ortega (1914) y desarrollándolas a partir de la visión antropológica de Marías (1970) resultan capitales para que cualquier joven, cualquier familia y generación, y la sociedad occidental superen los problemas más acuciantes de nuestro tiempo.

Solventes estudios internacionales dedicados a las personas jubiladas, como los llevados a cabo por los investigadores Whitehouse y por Young y Yanke, demuestran que la participación en un programa intergeneracional provoca una mejoría notable en las circunstancias psicológicas, físicas y sociales de los abuelos (Canedo, García y Pacheco, 2014). En el terreno psicológico las personas ancianas experimentan una mejora de su autoestima y también en su actitud a la hora de afrontar problemas de salud mental, como han confirmado las investigaciones de Whitehouse (2013) y de Young y Yanke (2011). En el plano físico y social, el mejor estado de estos abuelos viene motivado porque al tener más y mejores relaciones sociales en su día a día, se reducen los daños emocionales causados por el aislamiento y la soledad. De esta manera, la tristeza o la frustración que genera estar solo cuando no se quiere ni es la opción vital elegida, no aparece, y los ancianos se muestran joviales y con un tono vital fuerte como consecuencia de esa red de relaciones interpersonales y sociales que desarrollan con hijos, nietos, familiares de segundo grado, amigos o vecinos. Así lo atestiguan los estudios de campo que han llevado a cabo Belgrave (2011) y Ferguson (2013). Estos casi dos años de la pandemia de la COVID-19 está sirviendo para constatar lo hasta aquí dicho en cada vez más casos y con situaciones de mayor gravedad en todo el planeta. 
Cuando los abuelos o las abuelas sufren una enfermedad mental no solamente les afecta a ellos, sino también a sus familias. La comunicación intrafamiliar se ve afectada porque se producen problemas de compresión de las nuevas circunstancias. En ocasiones, se desencadenan patologías adicionales entre los familiares, como demuestran estudios sobre la materia, lo que a su vez genera un deterioro de la evolución del trastorno que sufre la persona anciana. Para dar respuestas y posibles soluciones a esas realidades se vienen desarrollando las terapias psicoeducativas familiares para mejorar el diagnóstico de la patología y para prevenir la aparición de otros trastornos en los familiares que se encargan de su atención y cuidado. Las medidas psicoeducativas más acertadas son: la formación en torno al trastorno que sufre el paciente; la incorporación de nuevos hábitos cotidianos que permitan una relación sana entre el paciente y sus familiares; el establecer unos límites con un espíritu constructivo para saber hasta dónde se puede llegar y que las nuevas normas de convivencia sean conocidas y aceptadas por todos los miembros de la familia; y el desarrollar unas redes sociales amplias de cooperación para que tanto el paciente como la familia no sufran el aislamiento. Las denominadas intervenciones mixtas, familiares y personalizadas están siendo muy útiles para solventar los casos de trastornos de la personalidad y en concreto del trastorno límite de personalidad (TLP) (Moya y Peláez, 2013).

Que se fortalezca la cohesión en la familia cuando se presentan situaciones como las descritas es clave para que la terapia familiar que se implemente dé los frutos esperados. Además, no solo supone un objetivo para las personas afectadas, sino que también hay que tener presentes los motivos y las expectativas que cada miembro de la familia tiene sobre la terapia dada la complejidad de las situaciones que se dan y la diversidad biográfica y de personalidad de cada uno de ellos (Escudero, 2009; citado en Moya y Peláez, 2013).

Los menores que también participan en estos programas intergeneracionales se ven notablemente beneficiados, ya que, además de comenzar a mirar a la vejez de sus mayores con otra perspectiva positiva, alejada de prejuicios derrotistas y tóxicos, les genera una mejora de su autoestima y de su sentido de la responsabilidad para consigo mismos y para con sus adultos. Educar y formar para superar prejuicios es otro reto que desde la escuela a la universidad, pasando por los institutos, se ha de instaurar en esta centuria, lo que repercute 
en favor de una mejor vertebración de la sociedad de la que son miembros las tres generaciones implicadas (O'Connor, 2012; Shedletsky, 2012). Otras circunstancias que se ven mejoradas son el absentismo escolar y el mantenerse alejados del consumo de drogas, lo que tiene un impacto, a su vez, en la mejora en el aprendizaje de nuevas aptitudes, competencias y habilidades, y en el fomento de la creatividad de los niños y adolescentes (Canedo, García y Pacheco, 2014).

\subsection{Educación alimentaria}

Se necesita implantar un programa formativo sobre nutrición y buenos hábitos alimenticios y de relación a lo largo de todo el sistema educativo, haciendo hincapié en el tránsito de la adolescencia a la edad adulta. Esta circunstancia, como la mayoría de las cuestiones, no pueden abordarse hoy en día solo desde un prisma local o nacional, sino que requieren y exigen respuestas inteligentes, consensuadas, coherentes y comprometidas a nivel de la Unión Europea y de Occidente. La sociedad europea y occidental que va más allá de la suma de las sociedades nacionales de cada país miembro, porque invoca a los usos y costumbres y a las circunstancias comunes que se comparten, como demostró Ortega y Gasset en los años veinte del siglo pasado en su célebre ensayo La rebelión de las masas, se hace más visible y presente en pleno siglo XXI (Ortega, 1986).

\subsection{Respuestas a los nacionalismos excluyentes y formación histórica}

Si la Unión Europea no promueve que los docentes en todas las etapas educativas formen a sus discentes contemplando no solo la historia nacional de cada país sino también cómo cada nación ha influido en la esfera internacional, se agravarán problemas como los nacionalismos y las posiciones de la ultraderecha o de la ultraizquierda que han emergido de nuevo en Europa y América en el último decenio 2008-2019, como consecuencia del estallido de la crisis económica mundial, de los errores cometidos por los partidos tradicionales que venían gobernando tanto en las naciones europeas como en 
el propio Parlamento Europeo, y de la pérdida de referentes éticos y morales, como ha demostrado la corrupción a nivel español, europeo y mundial.

He aquí otra de las circunstancias decisivas en la que nuestra juventud requiere de una sólida formación partiendo de un pensamiento histórico y crítico: educarse en la historia de su país, pero también de Europa y América, y hacerlo desde la lectura de los clásicos y extrayendo lecciones de vida que las conviertan en diques frente a cualquier intento de imposición de posturas incívicas, oligárquicas, partitocráticas o dictatoriales. Para formar en la tolerancia es imprescindible el conocimiento profundo de la historia, y su análisis a través de la filosofía, la ética y la moral, y el derecho. Pero para abordar esta circunstancia también hay que contar con la implicación de las familias, ya que en el seno de estas se adoptan posiciones sobre los nacionalismos. Si las familias no son conscientes del impacto que los nacionalismos tienen en la vida cotidiana de cualquier país o a nivel internacional, ni saben identificar a los líderes nacionalistas y lo que ellos y sus movimientos pretenden, habrá un vacío formativo de nuestros jóvenes. Hoy en día, se hace imprescindible que las familias y los menores sepan reconocer quién y cómo es un líder nacionalista y qué objetivos quieren imponer los grupos nacionalistas, así como los medios de comunicación y redes sociales que propagan sus mensajes por ser aliados de aquellos.

\subsection{El valor terapéutico de la narrativa}

Llegado a este punto, se hace necesario recurrir a la literatura y su labor terapéutica humana, ¿quién no ha disfrutado contándole un cuento a su hijo antes de dormir? Una vez más el mundo del cine nos puede ilustrar de forma clarividente para explicar el valor de la literatura para, por un lado, entender la historia, y, por otro, para sanar las heridas que los movimientos políticos causan en la persona, en la familia y en la sociedad. Un ejemplo es La vida es bella. En este film, un auténtico cuento audiovisual en formato cinematográfico sobre el drama del Holocausto durante la Segunda Guerra Mundial, se puede apreciar cómo el padre, Guido, crea una fábula para enmascarar a su hijo, Josué, el drama que están viviendo: estar encarcelados y bajo el yugo de poder ser asesinados en cualquier momento por ser judíos dentro del campo 
de concentración, así como la separación con la esposa y madre dentro del mismo campo de exterminio (Benigni, 1997).

La novela y el cuento aportan ese fomento del placer compartido entre personas de distintas generaciones. Además, la novela, siguiendo a Ortega, permite a la persona entender la vida humana, las relaciones humanas y cómo se tejen las relaciones sociales e institucionales (Ortega, 1914). Para Marías:

La novela tiene su génesis en las tragedias griegas, es la creación donde la historia personal fermenta. El problema de la novela griega es que ha sido corrompida cuando se le ha incorporado el mito, que es una realidad negativa o hipócrita. La novela es un conjunto de diálogos, de cambio de afectos de hombre a hombre (Marías, 1969: 200-201).

En los institutos mayoritariamente se enseña a pasar los exámenes de cada curso con la mira puesta en la EvAU (prueba de acceso a la universidad), pero no se detienen los profesores junto a los discentes a auscultar las biografías de los personajes, qué tipo de vida quisieron llevar, cómo eran las relaciones interpersonales o familiares en el tiempo narrado, qué pros y contras presentaban las instituciones públicas y privadas de la época descrita, ni tampoco se enseña a indagar en las cuestiones sociales determinantes. El proceso inverso rara vez se hace desde la Universidad, bajar a los centros de Secundaria, Bachillerato y FP para trabajar de la mano del profesorado y del alumnado de esa etapa previa, y crear sinergias en beneficio de todos, de la ciudadanía y del país.

Resulta paradójico que la narrativa a través de sus diferentes géneros dedica obras a la cuestión familiar y social, así como también los medios de comunicación y las redes sociales dedican buena parte de sus contenidos a la realidad de las familias y de la sociedad. Pero hoy en día, salvo honrosas excepciones, se tiende a hacerse de forma trivial, banal y sin entrar en un análisis profundo y sanador.

\subsection{Formación en análisis crítico de los medios de comunicación}

Como invoca el sociólogo y experto en medios de comunicación Juan Carlos Pérez (2021) en su reciente ensayo Ultrasaturados, las nuevas genera- 
ciones necesitan de una formación específica para gestionar la información, la desinformación, la manipulación o el exceso de opinión que generan a diario medios de comunicación y redes sociales. La formación en comunicación resulta decisiva para cualquier persona en el siglo XXI y posteriores.

Si nos centramos en la creación audiovisual y cinematográfica en los últimos cuarenta años, observamos asimismo cómo las productoras y canales de televisión y de cine vienen creando series y películas en el que el tema principal son las familias y sus circunstancias, así como la realidad social: podemos recordar desde clásicos de finales de los años setenta y principios de los ochenta como las series Con ocho basta o Los Roper, hasta todo un clásico del séptimo arte como Kramer contra Kramer. Más recientes son las series televisivas Breaking Bad, Modern Family o Cuéntame cómo pasó (Martínez y Crespo, 2017). E incluso el mundo de los dibujos animados centra su atención en la vida familiar y social a través de clásicos como Don Quijote de la Mancha; Marco, de los Apeninos a los Andes; Heidi; Los Picapiedra; o Los Diminutos. Tengamos presente que los dibujos animados son, por un lado, un recurso clave en la entrada de los menores en el mundo audiovisual y del cine. Un estudio muy reciente llevado a cabo por investigadores de la Universidad de Alicante muestra cómo el cómic es un recurso interdisciplinar de primera magnitud para formar un espíritu crítico en los jóvenes (Gómez y Ruiz, 2019). Esa investigación ha confirmado que el uso combinado del cómic y de las TIC mejora la predisposición de los discentes hacia los contenidos que adquirir. En este sentido, se pueden destacar clásicos del cómic llevados a la pantalla como Mickey Mouse, La familia del Pato Donald, o los más actuales Buscando a Nemo o Los Simpsons.

Y, por otro, cuando estos son de calidad, se convierten en un recurso educativo en valores, en actitudes y comportamientos, en creatividad y en inteligencia emocional. El cómic resulta muy estimulante desde hace décadas a cientos de miles de jóvenes lectores para adentrarles tanto en el mundo de la lectura, como en el del dibujo o en el fomento de su imaginación creativa. Es un vehículo adecuado para estimular a las nuevas generaciones a que primero se familiaricen y disfruten de la lectura de ese tipo de contenidos para que después estén preparados para leer obras maestras de la literatura o de cualquier otra materia. 


\subsection{Formación de padres y madres}

Por todo ello, se hace imprescindible que los padres reciban esa instrucción. Es una educación y formación intergeneracional, que no solo abarca el colegio, los institutos y a universidades, sino que ha de traspasar sus muros y paredes. Que esos dejen de ser compartimentos estancos como también lo son muchas familias desestructuradas y unas sociedades demasiado rígidas por las presiones de los grupos de interés y de presión. Y que unos y otros aprendan desde su posición a compartir una filosofía sustentada en ser vasos comunicantes frente a la actual viciada por la incomunicación, provocada por las trincheras y visiones de sus compartimentos estancos. Una formación en la que recordar al niño o a la niña que fueron es fundamental. Volver a su pasado para ayudar a construir el presente y el futuro de sus descendientes, de las nuevas generaciones. Volver a ponerse en la piel del menor que fueron para llevar a la práctica las buenas costumbres que sus mayores practicaron con ellos para vivir ratos de alegría, de intimidad, de comprensión y de intercambios de vivencias. Apostamos por que no solo se presente a la institución de la familia como en crisis, algo habitual en los medios de comunicación y en el mundo del cine hoy, sino también a bucear qué hay debajo de esa superficie para ver las otras nueve décimas partes del iceberg familiar. Desde la perspectiva de las investigadoras Martínez y Priego se hace imprescindible ir más allá de los estereotipos y de las imágenes habituales que de las familias se muestran a través de los medios de información: ellas hacen hincapié en "superar las barreras ante las que se encuentra una -no la única- de las notas esenciales de la institución familiar: el estar formada por un varón y una mujer «armónicamente» relacionados" (Martínez y Priego, 2017: 109).

Mutatis mutandis, lo dicho sobre los estereotipos vertidos sobre las familias podemos extrapolarlo sobre los grupos sociales, étnicos, los países, etc.

\subsection{Formación en educación emocional y sentimental}

El papel del hombre ha cambiado de manera sensible en las últimas décadas como consecuencia de los nuevos roles que la mujer asume (Marías, 1992: 1). 
Al igual que desde hace años se comparten las responsabilidades económicas y las tareas del hogar y de la educación de los hijos, el hombre tiene que dar un paso en la formación de su inteligencia emocional. Ello implica que hombres y mujeres, la sociedad y las instituciones, le quiten ese papel de fortaleza que a lo largo de la historia se le ha asignado, y que en parte ellos mismos asumieron. En el estudio de Robert Waldinger sobre la felicidad, llevado a cabo desde 1938 hasta 2013, se ha cuestionado a cientos de miles de personas sobre su trabajo, su vida familiar y su salud. Hoy en día siguen participando sesenta de los setecientos veinticuatro hombres que lo iniciaron. Las conclusiones más importantes de este trabajo es que "las buenas relaciones nos hacen más felices y más saludables" (Waldinger; citado en Rojas, 2018: 37). El sistema educativo en todo Occidente tiene un océano en esta realidad decisiva en las trayectorias de la persona y de los pueblos, y ha de afrontarla de forma coherente, constante y sabia a partir de este siglo. Se requiere educarnos en las emociones y en los sentimientos.

A las mujeres se les permite expresar sus emociones y sentimientos, incluso la transmisión del dolor o de la pena a través de ese desahogo tan sano que es llorar, pero a los hombres, no. Ha llegado la hora de que ese tabú social se supere, y de que el hombre muestre sus sentimientos y emociones, sobre todo cuando sufre, y que ello no sea visto ni por su pareja, ni por su familia ni por su entorno más cercano, ni por la sociedad, como una muestra negativa y de debilidad estructural. La superación de ese momento o de esa etapa dolorosa que vive un hombre adulto no solo va a ser un logro personal, sino también abrirá la vía para que su propio padre, el abuelo, supere traumas del pasado. $\mathrm{Y}$ que sus ejemplos sirvan a otros padres e hijos en similares o idénticas circunstancias.

Esto demuestra la aseveración de Ortega:

Este tipo de relación solo es posible con el individuo al que conocemos, con el prójimo o próximo. A esta capacidad de responderme en toda la amplitud de mis acciones, llamo corresponderme o reciprocarme. Pero si no hago más que estar abierto al otro, darme cuenta de que está ahí con su yo, su vida y su mundo propio, no hago nada con él y ese altruismo no es aún relación social. Para que ésta surja es menester que actúe o accione sobre él, que provoque en él una respuesta. Entonces, él y yo nos somos, y lo que cada uno hace 
respecto al otro es algo que pasa entre nosotros. La relación nosotros es la primera forma de relación social o socialidad (Ortega, 1957: 152).

Una circunstancia decisiva es cómo están evolucionando en un sentido u otro las relaciones entre abuelos, padres y nietos debido a los hábitos de vida de nuestro tiempo. Se dan situaciones entre los adultos que se pueden superar si todos los implicados ponen de su parte para hacerlo posible. Por ejemplo, cuando los pequeños reclaman a sus padres comer chucherías o ciertos alimentos, que previamente sus abuelos sí les han dado. $\mathrm{O}$ cuando los adolescentes buscan la complicidad de aquellos para llegar más tarde al hogar porque empiezan a salir y demás. Esto genera situaciones de tensión. Frente a ellas es necesario consensuar con antelación las decisiones que se toman para mandarles un mensaje común y coherente a los menores de edad. Y para que estos no se den cuenta de que ese tipo de coyunturas provoca fricciones entre sus mayores y acaben convirtiéndose, por un lado, en un campo minado para el chantaje emocional, $y$, por otro, en una tierra fértil para otras discusiones y desencuentros en la familia. En Occidente y en las sociedades con un alto poder adquisitivo en Asia (como Japón o los países del golfo Pérsico) se vive imbuidos por un materialismo excesivo y tóxico. Se ha olvidado el sentido de la vida, como recuerda la Dra. Marian Rojas siguiendo los estudios empíricos del psiquiatra Víctor Frankl (Rojas, 2018), y la filosofía de la razón vital e histórica de la Escuela de Madrid. Las consecuencias son nocivas para la vida tanto de las nuevas generaciones como de las generaciones de adultos y abuelos. Por ello, dada la socialización temprana que viven los menores desde su infancia y hasta cumplir la mayoría de edad, también se requiere que los docentes reciban una formación actualizada de esta realidad. Y que, durante toda la etapa educativa, sobre todo desde la Escuela Infantil hasta el Bachillerato o la FP, haya un feedback permanente entre los cabezas de familia y el profesorado. Desde el respeto a la intimidad de los menores y de la propia familia es imprescindible que haya, por un lado, un conocimiento profundo del estado de la comunicación intrafamiliar por parte de los profesores de los más pequeños. Y, por otro, que ese flujo comunicativo también vaya desde los docentes, el psicólogo y la Dirección del centro para saber cómo se comportan los niños y adolescentes en su día a día escolar (Daudinot, 2012). 
Desde marzo de 2018 grandes colectivos de personas, liderados por mujeres, se manifestaron en España y en otros países occidentales para reclamar el derecho a conciliar la circunstancia laboral con el resto de las circunstancias. Es insano continuar con ciertos usos o costumbres del pasado cuando las circunstancias de la mayoría de las personas han variado. Hacerlo es perpetuarse en el error y poner trabas a la vida personal y a la convivencia familiar; además, se dificultan las relaciones con las entidades en las que cualquiera trabaja. Todo ello influye notablemente en el desarrollo cotidiano de un país o de la Unión Europea. La educación cívica para que todos conciliemos la vida estudiantil y la vida profesional con la vida interpersonal y social no puede seguir siendo ignorada en el siglo XXI.

A lo largo del verano de 2018 se trasmitió de forma superficial a la opinión pública europea el debate en la UE de unificar los horarios entre los países miembros. Resulta curioso que una cuestión tan determinante como esta, que ha estado abierta a que cualquier persona pudiera participar para reflejar su posición, apenas se le haya dado cobertura en los medios de comunicación ni tampoco se haya publicitado de manera suficiente por parte de las instituciones comunitarias, con el propósito de fomentar una alta participación de la ciudadanía europea. Es un claro ejemplo de desconexión entre la realidad diaria que viven los 442 millones de personas que aproximadamente conforman la población comunitaria y las instituciones de la UE, así como del tratamiento superficial dado por los medios de comunicación a una cuestión clave. Un año después de que se lanzara la noticia, esta cuestión trascendental ha desaparecido de la agenda de los medios de comunicación, es una muestra clara del fenómeno que Noelle Neumann denominó "la espiral del silencio" (NoelleNeumann, 2010). Y, sin embargo, en la sociedad europea la conciliación de la vida personal y laboral es una cuestión decisiva que requiere de soluciones comprometidas, coherentes, inteligentes, responsables y honradas.

Resulta paradójico que desde Bruselas se quejen de la baja participación de los ciudadanos en las elecciones europeas, y que desde instituciones como el Instituto Ortega y Gasset desde finales de los años noventa se denunciase la falta de acervo democrático en los organismos europeos, incluido el propio Parlamento. También es contradictorio que desde ciertas fuerzas políticas en España y en el resto de Europa se hable hasta la saciedad de fomentar la demo- 
cracia participativa y que para una cuestión clave como esta de la unificación de los horarios no hayan fomentado la participación.

Junto a esa cuestión de los usos horarios, la formación en inteligencia emocional es cada día más prioritaria en la sociedad que vivimos. Los jóvenes la reclaman para mejorar la comunicación con sus padres (Carmona, $4 \mathrm{de}$ abril de 2018). Hay padres y madres, sobre todo estas, que fomentan más la conversación con sus hijos para saber cómo están, qué les ilusiona o qué les preocupa (Marías, 1993). Pero también hay otros que por diferentes motivos no promueven ese diálogo fundamental: desde los horarios profesionales de sus progenitores hasta la falta de esos hábitos comunicativos.

La emérita María Jesús Comellas, coordinadora del Grupo de Investigación, Orientación y Desarrollo (GRODE), incide en la necesidad de mantener la calma y la paciencia para comunicarnos con los menores. Es imprescindible, según ella, favorecer la comprensión, el respeto mutuo y no emitir juicios de valor. Siendo clave llevar a cabo con convicción los comportamientos que nos indican los pediatras o los profesores, no cediendo al chantaje emocional. La escuela y la familia han de conocerse y apoyarse, evitando las desconfianzas mutuas (Comellas, 2016). A la hora de relacionarnos con los niños hay que hacerlo con buen humor para romper con la situación de presión creada y evitar así explotar con un comentario duro o agresivo. Una vez superada esa coyuntura será bueno recordarla con ternura (Comellas, 2016). Cuando se le diga “¡no!” al menor, no hacerlo con malos modos. El buen humor y la buena actitud ayudarán a que lo entienda, a que haya feeling con él a pesar de no sentirse correspondido, y a que se le ofrezca otras vías mediante la intuición (Comellas, 2016).

En estos años se han consolidado los nuevos tipos de familias, por lo que todo lo dicho antes cobra mayor relevancia. Un estudio de Castells y Subirats indica que en el siglo xxi el $16 \%$ de la población mundial son matrimonios de toda la vida. E1 $24 \%$ son parejas fruto de una segunda relación. Y el $60 \%$ restante personas que no tienen una relación sentimental estable (Castells y Subirats, 2007). 


\section{§3. Resultados}

Que tanto en las familias que perduran, como en aquellas otras en las que el matrimonio se ha separado, o en las monoparentales, surgen distintos mensajes a los niños que con frecuencia les hace entrar en conflicto. Los pequeños ante una misma situación, por ejemplo, cómo comportarse en la mesa, se ven en la tesitura de elegir unos hábitos u otros diferentes e incluso a veces contradictorios. Tengamos presente que sobre todo en las edades más tempranas aprendemos por mimetismo, copiamos los comportamientos de nuestros mayores, sin pararnos a pensar si lo que hacemos y cómo lo hacemos está bien o no. Esas situaciones se agravan en matrimonios o parejas que se han separado o divorciado, y salvo honrosos casos, en ocasiones los adultos aprovechan esos contextos para seguir soterradamente librando su pugna por rencores hacia la expareja, no siendo conscientes de la gravedad que ello tiene para el crecimiento sano emocional del vástago común.

E incluso el diálogo cercano y sincero con familias que siguen unidas revela que hay casos en los que desavenencias no resueltas entre los dos miembros de la pareja, o incluso entre los cabezas de familia y los abuelos, generan un discurso y un comportamiento contradictorio hacia la prole. Esta se encuentra en medio, escuchando y viendo qué camino tomar, cuando visto desde fuera por un tercero con conocimiento de causa pueden darse casos en los que o bien las opciones planteadas por ambos sean erróneas o bien que en esa situación concreta sea la opción de uno de los mayores la correcta. Esto último tampoco puede desembocar en el fomento de una sensación permanente de superioridad sobre el otro miembro de la pareja (Marías, 1995).

También fruto de la nueva realidad humana, intergeneracional y social de hoy, nos encontramos con que no solo los padres y niños necesitan de ayuda para su día a día. Los abuelos la requieren, como demuestra la soledad que padecen aquellos y también muchos adultos que por diferentes motivos sufren el desapego de sus familias y amigos. La soledad es un problema mundial, sobre todo en Occidente, como reflejan los estudios solventes de la socióloga británica Marian Barnes, y el programa que ha puesto en marcha el franciscano fray Enrique Lista en Betanzos para dar una respuesta humana a este drama. Una sociedad que no cuida a sus mayores no se cuida a sí misma, y todas las 
personas antes o después lo seremos. Por tanto, hoy con nuestros actos y no actos estamos sembrando para el futuro. Si a los menores se les manda el mensaje de que no hay que preocuparse por sus abuelos, ni verlos con frecuencia, ni relacionarse con ellos, no solo se les privará de un rico pozo de vida para su crecimiento, sino que también se abonará el terreno para que sean más individualistas y egocéntricos en un mundo que lo es en demasía. Hoy, ante las circunstancias laborales de los padres hay que destacar la labor extraordinaria que están haciendo cientos de miles de abuelos cuidando a sus nietos, trayéndolos o llevándolos al colegio, y procurando compartir actividades que les gustan a aquellos. Y la aportación afectiva y económica de los abuelos a sus hijos y nietos durante los años más duros de la crisis (Carmona, 26 de julio de 2018).

\subsection{El caso de Guadalajara}

Las frecuentes visitas a la Biblioteca de Guadalajara, la observación del Maratón de Cuentos que se celebra cada mes de junio allí, el Ciclo Musical de Julio en los Jardines del Infantado, las ferias del mes de septiembre, entre otros eventos culturales y sociales, muestran que en esa ciudad hay un notable ejemplo de lo que es la convivencia intergeneracional sana. Educar en el fomento de las relaciones intergeneracionales es otra cuestión capital de nuestra época. Siguiendo a Ortega y Gasset, y Julián Marías, que demostraron que las dos terceras partes de toda filosofía se hace mirando, se han observado los comportamientos y usos de esos conciudadanos (Ortega, 1914). Y en esa mirada se ha apreciado la diferencia con otros lugares de España. Es cierto que Guadalajara es una urbe con una población de 87.484 habitantes y una provincia de 261.995 personas (Padrón, 2021); por tanto, su comparativa se enmarca en las ciudades y provincias de tamaño medio.

Solo hay que ir a esa biblioteca o a la Biblioteca José Luis Sampedro de Madrid cualquier día para ver cómo, por ejemplo, en la Hemeroteca leen la prensa diaria y las revistas una entrañable pareja de abuelos más cercanos a los noventa que a los ochenta años, mientras otros más jóvenes consultan el catálogo con la siempre atenta y profesional atención del personal de las bibliotecas. O cómo es posible en la primera planta que a la vez convivan un 
grupo de estudiantes con sus tareas escolares mientras otras personas preparan una oposición o alguien toca el piano para el deleite de la mayoría.

Lo habitual es que personas de distintas edades y generaciones participen con continuidad en la mayoría de los eventos educativos y culturales que se celebran en Guadalajara. En cambio, ese uso, esa costumbre no está establecida como costumbre general ni social en otras partes de España o del mundo: se constatan una vez más los compartimentos estancos. ¿Qué implican?

Que la costumbre mayoritaria establecida es que la gente vaya solo a lo que le interesa o le resulta más afín. Seguro que existen ese tipo de personas, pero no es lo que describe ni representa al cuerpo social que asiste a los eventos culturales organizados por las instituciones públicas y privadas de Guadalajara. Hay mezcolanza, la mayoría igual va al Maratón de Cuentos o a los Viernes de Cuentos de octubre a mayo, que al ciclo teatral del Teatro Moderno. O igual asiste a un concierto musical en los Jardines del Infantado que se reúne en la Plaza Mayor para ver a las compañías de teatro y músicos reencarnar una pieza para deleite de todos. Se producen los injertos (Marías, 1993), que permiten evitar los compartimentos estancos que Ortega denunció y demostró que se habían formado en España y que explicaban su desvertebración (Ortega, 1921). Para Marías (1993: 106), el injerto

introduce en el otro algo propio, pero siguen siendo lo que eran, modificado. Si ese influjo es benéfico, sobre todo en el sentido de estimular y reforzar lo que el otro auténticamente es, se puede decir que es liberador; si se ejerce en sentido contrario, si aparta de sí mismo al que lo recibe, es el ejemplo máximo de corrupción.

En cambio, los compartimentos estancos son como las aguas estancadas, están corrompidas, no son saludables. No permiten ni ser bebidas ni usadas para el consumo humano, ni para regar las tierras, ni tampoco permiten la convivencia sana entre sus miembros. Los compartimentos estancos son una manifestación palpable de otras conductas humanas egocéntricas que entre otras consecuencias tiene el desarrollo y mantenimiento de los nacionalismos.

Es obvio que se requiere una formación académica para todos los grupos implicados. La observación de la realidad cotidiana nos lleva a plantear hasta 
qué punto la formación tal y como está diseñada desde hace décadas y hasta nuestro tiempo, en exceso concebida para las etapas de la vida antes de ingresar en el mundo profesional, nos está llevando a un cierto distanciamiento no solo en los contenidos que se abordan, sino sobre todo a una falta de conexión entre padres, abuelos y nietos.

Aunque padres y madres estén pendientes de la educación y formación de sus hijos a través del seguimiento que hacen con los profesores en las tutorías, y en el hogar comprobando si hacen los deberes y estudian. A pesar de que los abuelos también en numerosas ocasiones colaboran llevando o trayendo a sus nietos a la escuela, e incluso también realizando esas tareas escolares con aquellos, las experiencias propias y ajenas nos llevan a afirmar que hoy hay un salto entre generaciones que es muy significativo.

Cuando están en los cursos previos a la universidad y en el primer año universitario, ellos, como cualquier generación en el pasado, requieren que se les oriente con seriedad y amplitud de miras sobre los múltiples caminos que pueden tomar. Y, en especial, que sean sinceros sobre todo consigo mismos y también con sus familias para que sean libres y valientes y elijan aquel camino que les guste, por el que sientan auténtica vocación. Esta es una cuestión pendiente en España desde hace al menos seis décadas, pero también se ha agravado en el resto del mundo, sobre todo en Occidente, desde los años setenta. El mensaje predominante que se lanza desde muchos medios de comunicación a la sociedad y también desde las instituciones políticas, desde las empresas y muchas universidades, es elegir los estudios en función de las supuestas salidas laborales que estos tienen. Resulta interesante el estudio que se llevó a cabo sobre este tema en la Universidad de Córdoba (Amor y Serrano, 2016).

Bajo ese discurso que llevamos oyendo y escuchando desde los años noventa, hay toda una ideología que busca marcar y controlar los hábitos de vida y hasta el día a día de cualquier persona y familia: el mercado laboral ofrece a través de sus empresas e instituciones públicas una serie de puestos de trabajo que necesitan ser cubiertos para que este siga produciendo $\mathrm{u}$ ofreciendo determinados servicios. La formación de esos futuros profesionales en la mayoría de las ocasiones se hace a través de las universidades e institutos de FP. Los medios de comunicación se convierten en altavoces propagandísticos de unos y otros haciendo clasificaciones de cuáles son los trabajadores más demandados 
y las llamadas carreras del futuro. Así, se ha entrado en un círculo vicioso en el que buena parte de la sociedad y, por tanto, de las familias, están haciendo el juego a aquellos (Sennett, 2000). Ante el miedo al desempleo, a no encontrar un puesto de trabajo, no se tiene en cuenta ni la ilusión de la persona joven por unos determinados estudios o formación, ni tampoco el que innumerables oficios aún necesarios y con una riqueza cultural enorme se hayan abandonado. Tampoco se tiene presente que, como la historia nos demuestra a lo largo de esta, se han ido creando nuevos estudios y profesiones demandadas por las propias necesidades de los seres humanos y de la sociedad.

Las relaciones intergeneracionales son situaciones idóneas para abordar esas realidades mientras se está viendo una película o tras concluir esta; o bien antes de irse a dormir. Durante esos ratos, según Comellas, hay que "evitar preguntarles en exceso por las responsabilidades, y recordarles cuánto se les quiere de manera afectuosa. Se ha de estar atento a cualquier mirada o petición" (Comellas, 2016: 62). Y es bueno recurrir a casos iguales o similares que vivimos a esa edad que ellos tienen ahora.

Otro momento y espacio oportuno para fomentar las buenas relaciones entre padres e hijos a esas edades es propiciar "ratos de encuentros con sus amigos en casa, y conversar de manera cercana y cariñosa sin que tengan en ningún momento la sensación de sentirse controlados" (Comellas, 2016: 63-64).

Los momentos compartidos alrededor de la buena literatura son magníficos para fomentar esas relaciones intergeneracionales. El cuentista Pep Bruno señala que sus hijos ya adolescentes no se imaginan una Guadalajara sin su Maratón de Cuentos. Aquel nació fruto de la vocación de los miembros del Seminario de Literatura Infantil y Juvenil por el cuento, y de la decisión valiente y constante por llevarlo adelante, hasta convertirlo en un referente a nivel internacional, cuya próxima estación pudiera ser la Declaración por la Unesco de Guadalajara como provincia del cuento en España. Esa transmisión oral de la sabiduría popular y científica se necesita para mejorar la comunicación intrafamiliar. El estilo, las maneras y la actitud ilusionada que implica contar un cuento es un método muy estimulante para que padres, abuelos y nietos sean capaces de ponerse en la situación del otro cuando las circunstancias lo requieran. A los menores les resulta muy grato que el adulto y ellos encarnen cada uno a un personaje del cuento. Eso les genera risas, complicidades, los 
llevan a compartir ese rato, a intercambiar ideas y contenidos, y los predispone para compartir historias, dudas y sentimientos (Bruno, 2018; citado en Carmona, junio de 2018).

\section{$\S 4$. ConCLusiones}

Resumiendo lo dicho, “¿qué pretendemos con ello? ¿Jóvenes con buenas notas o personas maduras y seguras?" (Comellas, 2016: 41).

En primer lugar, se aunará la experiencia y competencia interdisciplinar de los profesionales con la voluntad, la ilusión y la experiencia vital de los abuelos. Así será más probable alcanzar los resultados esperados, evitando que los cambios de hábitos de vida y sugerencias de los abuelos sean vistos como que nacen de ellos o de su capricho, cuando en realidad radican de los conocimientos de esos profesionales con años de experiencia en atender casos similares, y que a su vez tienen la humildad de escuchar a sus pacientes. Asimismo, se abre la posibilidad de superar uno de los prejuicios que hoy en día aún permanecen en amplios grupos sociales, como es ver a la figura del psicólogo con recelo.

En segundo lugar, para responder a cómo hay que mejorar los hábitos educativos, de socialización, así como los alimenticios para superar los problemas de obesidad o desnutrición (anorexia o bulimia), y también enriquecer los hábitos de relación y comunicación entre padres e hijos, esos profesionales de diferentes campos les van a aportar a los séniores una serie de recursos de actuación y métodos para que, con el ejemplo de vida, esas madres y esos padres que ahora tienen que reconstruir la vida familiar tengan a su vez ejemplos de buenas prácticas de los que aprender.

En tercer lugar, cabe convertir ese proceso de formación de los voluntarios sénior en una especie de laboratorio de buenas prácticas que se transmiten y se ponen en uso para solucionar esos problemas. Pero también para generar un feedback: los séniores a su vez con el paso del tiempo han de comunicar cómo va evolucionando el entorno familiar. ¿Cómo mejora? ¿En qué aspectos y circunstancias? ¿Qué puntos de discordia se producen aún, qué nuevas situaciones se dan? Y esas realidades que observen serán transmitidas a esos 
profesionales que los formaron (profesores, psicólogos, médicos, sociólogos, educadores y comunicadores) para saber el estado real de la cuestión una vez transcurrido un tiempo de referencia y actuación.

Junto a las competencias y los saberes acumulados a lo largo de la vida, es necesario que estas personas reciban una formación complementaria para ser entrenadores de madres que tienen problemas en la reconstrucción de la vida familiar. Esa formación complementaria habrá de ser interdisciplinar por parte de psicólogos, sociólogos, nutricionistas, docentes y comunicadores.

Para rematar este artículo, hay que señalar que uno de los problemas de nuestra época es el excesivo tiempo y esfuerzo, y la desmesurada valoración que se le da a lo laboral. No se trata de restarle importancia ni trascendencia en la vida de una persona, de una familia o a nivel social. Pero sí que no ocupe el centro de nuestras existencias ni conversaciones. Si seguimos dándole tan excesiva repercusión, el tiempo y el interés por las siguientes circunstancias de la vida se ven mermados. Es clave disponer de tiempo y ganas para ocuparnos de la pareja, de la familia, de los amigos, de leer un libro, de escuchar música, de la meditación, de disfrutar de la naturaleza, del descanso, etc. Si la sociedad europea y occidental son capaces de llevarlo a cabo, sus sociedades, instituciones y países se beneficiarán.

Es necesario plantear, primero, las necesidades principales que tienen las familias y los menores con estos problemas de formación y comunicativos y, a continuación, cómo es posible ayudarles para afrontar esa realidad con una formación competente y comprometida con sus circunstancias. Se requiere la participación de aquellos que quieran ser entrenadores de las madres y de los padres que tienen dificultades con sus hijos adolescentes. Es también un método de fomento de las relaciones intergeneracionales. Por lo tanto, aunamos método e hipótesis para dar respuesta a la situación planteada.

Cualquiera ha experimentado en momentos de su vida esa necesidad de vivir aquello que le ilusionaba. Ahora, siendo capaces de mirar a ese pasado con sinceridad, se puede hacer un balance vital de aquellas experiencias que merecieron la pena ser vividas. Y cuáles se pudieron haber evitado de haber escuchado los consejos y el conocimiento que regala alguno de los mayores. Cabe la posibilidad así de que se reduzca el porcentaje de errores y de lamentos que conllevan las experiencias o los proyectos que nunca se deberían haber 
afrontado porque al final los frutos y las vivencias fueron negativos. Lo habitual entre nuestra juventud universitaria, cuando se les escucha en clase tras preguntarles si alguien les orientó en sus institutos o en sus casas sobre qué camino tomar después del instituto, es oír como respuesta mayoritaria no, salvo ejemplares excepciones. Y la respuesta la completan diciendo que esa etapa educativa está centrada en contenidos acumulativos para aprobar exámenes y llegar a la universidad tras una nota de corte. No se les enseña para que cada joven descubra quién quiere ser, cómo desea vivir o a qué quiere dedicar su vida. ¿Cómo son sus relaciones interpersonales o sociales? ¿Con qué situaciones se encuentran cuando tratan de establecer una comunicación con el personal de una institución pública o privada, y cómo gestionarlas?

Por ejemplo, como recuerda la experta María Jesús Comellas, "cuando ocurre un accidente doméstico pequeño hay que evitar la censura, y recordar que también es muy posible que nos pasara de niños" (Comellas, 2016: 45). Hay que ser comprensivos, no somos infalibles ni perfectos. No hay que caer en situaciones difíciles y trascendentales, y se han de imaginar escenas alegres y divertidas (Comellas, 2016). En esta época mucha gente entra en depresión o en estados de angustia y ansiedad porque no es consciente de qué es la felicidad hasta que la pierde. La persona se pierde en elucubraciones y mira a su alrededor y todo lo ve negro, el lado oscuro o negativo predomina en su cotidianidad. Por eso los expertos en psiquiatría y psicología están trabajando para que cualquier ser humano que llega a su consulta se dé cuenta de que la biografía de cada uno encierra etapas de derrotas y otras épocas de victorias, de luces y de sombras, de certezas y de incertidumbres. Por lo tanto, hoy en día y en el futuro se ha de aprender a encajar los golpes en los momentos complicados y exigentes para ser capaz de solventarlos y de reemprender el camino de la alegría cotidiana y de los aciertos (Rojas, 2018).

Esto último no es baladí, y menos en la sociedad actual, en la que muchos son los padrinos de los que triunfan, pero muy pocos los que se solidarizan con la persona que ha fracasado en un proyecto. Como señala Adela Cortina (10 mayo de 2017), la aporofobia, que es el rechazo que se ejerce sobre quien vive en la pobreza o es excluido por otro motivo, no se practica sobre el extranjero, sino sobre aquella persona nacional o no que vive sumida en la indigencia o 
con grandes dificultades para afrontar su día a día. La persona angustiada o sin éxito social es alguien que sufre la aporofobia en su cotidianidad.

En ese escenario descrito, los abuelos y abuelas adquieren un papel decisivo. Cualquiera que se haya detenido a observar y conversar con ellos antes y durante su jubilación se percata de que necesitan dotar de estímulos su día a día. Como a cualquier otra persona se les presenta cada jornada con sus 24 horas. En España, según la Dra. Belando-Montoro, a pesar del aumento notable de la proporción de población de mayores de 65 años en las últimas décadas, no se ha comunicado bien a la sociedad civil qué implicaciones y consecuencias tiene ser mayor. Desde su óptica existe en el imaginario colectivo de la sociedad española una visión negativa de los abuelos (Belando-Montoro, 2015). Por tanto, se hace necesario explicar las circunstancias que han provocado que haya arraigado esa visión negativa de la última etapa de la vida de las personas entre nuestros conciudadanos. Y además resulta clave darles respuestas sabias y equilibradas para que hombres y mujeres de España de las diferentes generaciones sean capaces de crear una sociedad intergeneracional a partir del aprendizaje de todas las generaciones implicadas en este proceso humano y social. El mañana no está escrito, pero en función de los caminos que se recorran y de los valores y decisiones que se adopten, la historia tomará un sendero u otro bien distinto.

Cuando las personas se jubilan, la vida adquiere una nueva perspectiva. El tiempo que se dedicaba antes a la labor profesional queda disponible para que la persona elija qué hacer con él. Eso abre la vía a contar con hombres y mujeres a quienes les ilusione realizar una labor tan importante como la aquí planteada. También hay adultos que, aun trabajando, por motivos de conciencia humana y cívica, deciden dedicar un tiempo de su vida a esas acciones u otras vinculadas con el tercer sector.

En primer lugar, ellos tienen una gran experiencia de vida acumulada en el trato con los niños y adolescentes. Antes de ser abuelos, han sido padres y madres. La perspectiva de los abuelos cuando se ha madurado como persona ofrece a los adolescentes una posición cercana y dialogante para hacerles ver qué hábitos han de cambiar; por ejemplo, cómo se alimenta. O cómo se relacionan en la mesa con sus familiares y otros. $\mathrm{O}$ si participan o no en la puesta de la mesa, y en la recogida de esta y de la cocina. 
En segundo lugar, esa sapiencia de vida puede ayudar a que la comunicación intrafamiliar sea más fluida. Los niños y adolescentes no ven a los abuelos como a los padres, estos últimos desempeñan un control más fuerte sobre sus hijos en las situaciones citadas: alimentación, hábitos en la mesa y quehaceres domésticos.

En tercer lugar, ese talante de los séniores puede abrir los cauces para que los menores de edad se den cuenta de manera más abierta de que esas enseñanzas de hábitos de vida en familia son importantes para su presente y su futuro. Ellos serán adultos algún día y tendrán que hacer la compra, cocinar, organizar un hogar y dar un espíritu sano a las relaciones familiares tejidas en torno a la mesa. Es una cultura de vida, por tanto, que hay que continuar.

En cuarto lugar, desde hace años se están perdiendo los buenos hábitos de comunicación y relación entre las diferentes generaciones a la mesa a la hora de las comidas y en otros contextos. En los años setenta y hasta principios de los ochenta del siglo xx era normal que la televisión no se pusiera hasta que no se había terminado de almorzar o cenar, y se recogieran la mesa y la cocina. Solo había una televisión y dos canales televisivos en un país como España (Pérez, 2021). Hoy en día, detectamos dos problemas: el primer escollo es más antiguo, que la televisión esté puesta mientras se come. Esto corta la comunicación entre hijos y padres. A mediados de los años cincuenta, el filósofo Julián Marías (1956) apuntó algunos de esos problemas que estaba generando el uso de la televisión en determinados momentos del día, fruto de su observación de la sociedad estadounidense.

Además, muchos abuelos se han incorporado a las nuevas tecnologías y usan el móvil y el WhatsApp, pero sin que interfieran en sus horarios de comidas. Por tanto, conservar esa buena costumbre de no usar los móviles durante las comidas familiares es otro valor importante que pueden aportar a sus hijos y nietos, es un logro social clave que se puede conseguir. Comellas aporta una solución inteligente para toda la familia: "dejar todos los móviles de la familia en una caja en el pasillo" (Comellas, 2016: 50). Si, por el contrario, queremos buscar un lado positivo al uso de las nuevas tecnologías y de sus aplicaciones de última generación, los investigadores Ghosh, Ratan, Lindeman y Steinmetz hablan de las posibilidades que aquellas ofrecen para dar respuestas inteligentes y saludables al envejecimiento conectado. Las nuevas tecnologías basadas en 
internet y en los dispositivos móviles abren todo un abanico de oportunidades tanto para los abuelos como para sus cuidadores frente a las necesidades educativas, sanitarias, sociales y funcionales que presentan, y conectándoles con familiares, amigos y con su comunidad (Canedo, García y Pacheco, 2014).

\section{REFERENCIAS BIBLIOGRÁFICAS}

AECOSAN (2015). Estudio Aladino. Estudio de Vigilancia del Crecimiento, Alimentación, Actividad Física, Desarrollo Infantil y Obesidad en España. Disponible en: $<$ https://acortar.link/WzpfNx>.

Amor, M. ${ }^{a}$ I., Serrano, R. (2016). El asesor académico: un nuevo perfil para la orientación y la tutoría en la universidad. Orientación y Sociedad (16), 15-23. Disponible en: $<$ https://revistas.unlp.edu.ar/OrientacionYSociedad/ issue/view/597/263>.

Belando-Montoro, M. ${ }^{\mathrm{a}}$ R. (2015). Conflicto intergeneracional y algunos caminos hacia la sociedad intergeneracional. Prisma social (14), 545-573. Disponible en: $<$ https://dialnet.unirioja.es/servlet/articulo?codigo $=6196779>$.

Belgrave, M. (2011). The Effect of a Music Therapy Intergenerational Program on Children and Older Adults' Intergenerational Interactions, Cross-Age Attitudes, and Older Adults' Psychosocial Well- Being. Journal of music therapy 48(4), 486-508.

Benigni, R. (director y guionista) (1997). La vida es bella. Melampo Cinematográfica.

Canedo, A., García, J., Pacheco, D. I. (2014). Educación intergeneracional de forma virtual. INFAD Revista de Psicología 1(3), 345-352. Disponible en: $<\mathrm{http}$ ://dx.doi.org/10.17060/ijodaep.2014.n1.v3.512>.

Carmona, M. (2020). Ortega y Marías, la filosofía del siglo XXI. Ed. Dykinson.

Carmona, M. (4 de abril de 2018). Así afectan los horarios de los mayores a los niños. El País. Disponible en: <https://elpais.com/elpais/2018/04/05/ mamas_papas/1522918568_682144.html>.

Carmona, M. (4 de mayo de 2018). Estas son las claves para reducir la obesidad en niños y adolescentes. El País. 
Carmona, M. (8 de junio de 2018). El profesorado no entiende al bachiller. Sí, debemos estudiar, pero tiene que ser compatible con vivir. El País.

Carmona, M. (26 de julio de 2018). Tus nietos se encariñan contigo tal y como eres. Ser abuelo te hace más altruista, menos egoísta. El País.

Carmona, M. (octubre de 2018). Maratón de cuentos. La terapia del arte. Disponible en: $<$ http://laterapiadelarte.com/numeros-de-la-revista/numero-37/ reportaje/maraton-de-cuentos/>.

Castells, M., Subirats, M. (2007). Mujeres y hombres, ¿un amor imposible? Alianza.

Comellas, M. J. (2016). Educar no es tan dificil como creemos. Lectio Ediciones.

Comisión Europea (2013). Educación abierta: fomentar el uso de las nuevas tecnologías desde la escuela y para toda la comunidad educativa. Disponible en: <http://goo.gl/AjlwQn>.

Cortina, A. (10 de mayo de 2017). Lo que molesta de los inmigrantes es que sean pobres. El País. Disponible en: < https://elpais.com/cultura/2017/05/08/ actualidad/1494264276_545094.html>.

Daudinot, J. (2012). La comunicación intrafamiliar: una necesidad en la formación inicial del maestro primario. Revista EduSol (40), 44-52. Disponible en: <http://www.redalyc.org/pdf/4757/475748679005.pdf >.

Directorate-General for Communications Networks, Content and Technology (2016). Pilot project for developing the use of new technologies and digital tools in education - Technologies and tools for children and young people with attention - deficit hyperactivity disorder (ADHD). Bruselas.

Escudero, V. (2009). La creación de la alianza terapéutica en la Terapia Familiar. Apuntes de psicología 27(2-3), 247-259.

Ferguson, L. (2012). Tackling loneliness in older age why we need action by all ages. Quality in Ageing and Older Adults 13(4), 264-269.

Gómez, I. M. a, Ruiz, M. (2019). El cómic como recurso didáctico interdisciplinar. Tebeosfera: Cultura Gráfica (10). Disponible en: <https://acortar. link/8JWdss>. 
Lindo, E. (16 de septiembre de 2018). Brote de titulitis. El País. Disponible en: $<$ https://acortar.link/4bmAAv>.

Marías, J. (1956). Los Estados Unidos en escorzo. Emecé Editores.

Marías, J. (1961). El método histórico de las generaciones. Alianza Editorial.

Marías, J. (1969). La imagen de la vida humana. En Obras, T. 5. Revista de Occidente.

Marías, J. (1986). La mujer y su sombra. Alianza Editorial.

Marías, J. (1992). La educación sentimental. Alianza Editorial.

Marías, J. (1993). Mapa del mundo personal. Alianza Editorial.

Marías, J. (1995). Tratado de lo mejor. Alianza Editorial.

Marías, J. (1996). Persona. Alianza Editorial.

Martínez, C., Crespo, M. ${ }^{\text {a }}$ G. (2017). ¿Poder o gratuidad? Aproximación sistémica para comunicar las relaciones mujer-varón. Revista de Comunicación 16(2), 108-122. Disponible en: <https://revistadecomunicacion.com/article/ view/989>.

Noelle-Neumann, E. (2010). La espiral del silencio: Opinión pública: nuestra piel social. Paidós Comunicación.

O'Connor, M. (2012). A Terror management Perspective on young Adults' Ageism and Attitudes Toward Dementia. Educational Gerontology 38(9), 627-643. doi: 10.1080/03601277.2011.595335.

Ortega y Gasset, J. (1914). Meditaciones del Quijote. Revista de Occidente.

Ortega y Gasset, J. (1921). España invertebrada. Revista de Occidente.

Ortega y Gasset, J. (1929). ¿Qué es filosofía? Revista de Occidente.

Ortega y Gasset, J. (1957). El hombre y la gente. Revista de Occidente.

Ortega y Gasset, J. (1986). La rebelión de las masas (25. ed.). Espasa Calpe.

Padrón (2021). Población de la ciudad y de la provincia de Guadalajara. Disponible en: $<$ https://padron.com.es/guadalajara/>.

Peña, B., Pezzoli, S. (2018). Propuesta de comunicación intergeneracional entre tercera edad y jóvenes en la sociedad del conocimiento. Revista de Comunicación de la SEECI (45), 1-14. doi: http://doi.org/10.15198/seeci.2018.45.01-14 
Pérez, J. C. (2021). Ultrasaturados: el malestar en la cultura de las pantallas. Plaza y Valdés.

Rodríguez, L., Peláez, J. C. (2013). Terapia familiar en los trastornos de personalidad. Acción psicológica 10(1), 75-84. Disponible en: $<$ https://www. redalyc.org/articulo.oa?id=344030771008>.

Rojas, M. (2018). Cómo hacer que te pasen cosas buenas. Espasa Libros.

Sennett, R. (2000). La corrosión del carácter: las consecuencias personales del trabajo en el nuevo capitalismo. Anagrama.

Shedletsky, L. (2012). Undergraduates Mentoring Older Adults: Breaking Stereotypes. Journal of Intergenerational Relationships 10(4), 400-414. doi: 10.1080/15350770.2012.726599.

Young, T. L., Janke, M. (2011). Older Adults' Perceived Benefits and Concerns in an Intergenerational Program: the Linc Project. Gerontologist (51), 600600 .

Whitehouse, P. (2013). The Challenges of Cognitive Aging: Integrating Approaches from Neuroscience to Intergenerational Relationships. Journal of Intergenerational Relationships 11(2), 105-117. Disponible en: $<$ https://doi. org/10.1080/15350770.2013.782740>. 